\title{
On cubic Hodge integrals and random matrices
}

\author{
Boris Dubrovin, Di Yang \\ SISSA, via Bonomea 265, Trieste 34136, Italy
}

\begin{abstract}
A conjectural relationship between the GUE partition function with even couplings and certain special cubic Hodge integrals over the moduli spaces of stable algebraic curves is under consideration.
\end{abstract}

\section{Contents}

1 Introduction $\quad 1$

1.1 Cubic Hodge partition function . . . . . . . . . . . . . . . . 1

1.2 GUE partition function with even couplings . . . . . . . . . . . . . . . 3

1.3 From cubic Hodge integrals to random matrices. Main Conjecture. . . . . . . . . . . . 4

1.4 Computational aspects of the Main Conjecture: how do we verify it? . . . . . . . . . 4

1.5 An explicit expression for $F_{g} \ldots \ldots \ldots \ldots \ldots \ldots$

2 GUE free energy with even valencies $r$

2.1 Calculating the GUE free energy from Frobenius manifold of $\mathbb{P}^{1}$ topological $\sigma$-model . 8

2.2 Proof of Prop. 1.4.1, Thm.1.4.3 . . . . . . . . . . . . . . . . . . 10

3 Verification of the Main Conjecture for low genera $\quad 10$

3.1 Genus $0 \ldots \ldots \ldots \ldots \ldots \ldots \ldots \ldots \ldots \ldots$

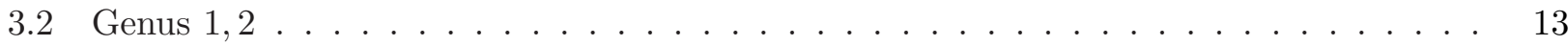

3.3 Genus $3,4 \ldots \ldots \ldots \ldots \ldots \ldots \ldots \ldots \ldots \ldots$

$\begin{array}{lr}\text { A Explicit formula for } F_{5} & 15\end{array}$

\section{Introduction}

\subsection{Cubic Hodge partition function}

Let $\overline{\mathcal{M}}_{g, k}$ denote the Deligne-Mumford moduli space of stable curves of genus $g$ with $k$ distinct marked points. Denote by $\mathcal{L}_{i}$ the $i^{\text {th }}$ tautological line bundle over $\overline{\mathcal{M}}_{g, k}$, and $\mathbb{E}_{g, k}$ the rank $g$ Hodge bundle. 
Let $\psi_{i}:=c_{1}\left(\mathcal{L}_{i}\right), i=1, \ldots, k$, and let $\lambda_{i}:=c_{i}\left(\mathbb{E}_{g, k}\right), i=0, \ldots, g$. Recall that the Hodge integrals over $\overline{\mathcal{M}}_{g, k}$, aka the intersection numbers of $\psi$ - and $\lambda$-classes, are integrals of the form

$$
\int_{\overline{\mathcal{M}}_{g, k}} \psi_{1}^{i_{1}} \cdots \psi_{k}^{i_{k}} \cdot \lambda_{1}^{j_{1}} \cdots \lambda_{g}^{j_{g}}, \quad i_{1}, \ldots, i_{k}, j_{1}, \ldots, j_{g} \geq 0 .
$$

Note that the dimension-degree matching implies that the above integrals vanish unless

$$
3 g-3+k=\left(i_{1}+i_{2}+\cdots+i_{k}\right)+\left(j_{1}+2 j_{2}+3 j_{3}+\cdots+g j_{g}\right) .
$$

The particular case of cubic Hodge integrals of the form

$$
\int_{\overline{\mathcal{M}}_{g, k}} \Lambda_{g}(p) \Lambda_{g}(q) \Lambda_{g}(r) \psi_{1}^{i_{1}} \cdots \psi_{k}^{i_{k}}, \quad \frac{1}{p}+\frac{1}{q}+\frac{1}{r}=0
$$

was intensively studied after the formulation of the celebrated R. Gopakumar-M. Mariño-C. Vafa conjecture $[17,24]$ regarding the Chern-Simons/string duality. Here we denote

$$
\Lambda_{g}(z)=\sum_{i=0}^{g} \lambda_{i} z^{i}
$$

the Chern polynomial of $\mathbb{E}_{g, k}$. A remarkable expression for the cubic Hodge integrals of the form

$$
\int_{\overline{\mathcal{M}}_{g, k}} \frac{\Lambda_{g}(p) \Lambda_{g}(q) \Lambda_{g}(r)}{\left(1-x_{1} \psi_{1}\right) \ldots\left(1-x_{k} \psi_{k}\right)}, \quad k \geq 0
$$

conjectured in [24] was proven in [18, 25]; for more about cubic Hodge integrals see in the subsequent papers $[19,20,28,12]$.

In the present paper we will deal with the specific case of Hodge integrals (1.1.1) with a pair of equal parameters among $p, q, r$; without loss of generality one can assume that $p=q=-1, r=1 / 2$. So, the special cubic Hodge integrals of the form

$$
\int_{\overline{\mathcal{M}}_{g, k}} \Lambda_{g}(-1) \Lambda_{g}(-1) \Lambda_{g}\left(\frac{1}{2}\right) \psi_{1}^{i_{1}} \cdots \psi_{k}^{i_{k}}
$$

will be considered. Denote

$$
\mathcal{H}(\mathbf{t} ; \epsilon)=\sum_{g \geq 0} \epsilon^{2 g-2} \sum_{k \geq 0} \frac{1}{k !} \sum_{i_{1}, \ldots, i_{k} \geq 0} t_{i_{1}} \cdots t_{i_{k}} \int_{\overline{\mathcal{M}}_{g, k}} \Lambda_{g}(-1) \Lambda_{g}(-1) \Lambda_{g}\left(\frac{1}{2}\right) \psi_{1}^{i_{1}} \cdots \psi_{k}^{i_{k}}
$$

the generating function of these integrals. Here and below $\mathbf{t}=\left(t_{0}, t_{1}, \ldots\right)$ are independent variables, $\epsilon$ is a parameter. The exponential $e^{\mathcal{H}}=: Z_{\mathbb{E}}$ is called the cubic Hodge partition function while $\mathcal{H}(\mathbf{t} ; \epsilon)$ is the cubic Hodge free energy. It can be written in the form of genus expansion

$$
\mathcal{H}(\mathbf{t} ; \epsilon)=\sum_{g \geq 0} \epsilon^{2 g-2} \mathcal{H}_{g}(\mathbf{t})
$$

where $\mathcal{H}_{g}(\mathbf{t})$ is called the genus $g$ part of the cubic Hodge free energy, $g \geq 0$. Clearly $\mathcal{H}_{0}(\mathbf{t})$ coincides with the Witten-Kontsevich generating function of genus zero intersection numbers of $\psi$-classes

$$
\mathcal{H}_{0}(\mathbf{t})=\sum_{k \geq 0} \frac{1}{k !} \sum_{i_{1}, \ldots, i_{k} \geq 0} t_{i_{1}} \cdots t_{i_{k}} \int_{\overline{\mathcal{M}}_{0, k}} \psi_{1}^{i_{1}} \cdots \psi_{k}^{i_{k}}=\sum_{k \geq 3} \frac{1}{k(k-1)(k-2)} \sum_{i_{1}+\cdots+i_{k}=k-3} \frac{t_{i_{1}}}{i_{1} !} \cdots \frac{t_{i_{k}}}{i_{k} !} .
$$

We note that an efficient algorithm for computing $\mathcal{H}_{g}(\mathbf{t}), g \geq 1$ was recently proposed in [12]. 


\subsection{GUE partition function with even couplings}

Let $\mathcal{H}(N)$ denote the space of $N \times N$ Hermitean matrices. Denote

$$
d M=\prod_{i=1}^{N} d M_{i i} \prod_{i<j} d \operatorname{Re} M_{i j} d \operatorname{Im} M_{i j}
$$

the standard unitary invariant volume element on $\mathcal{H}(N)$. The most studied Hermitean random matrix model is governed by the following GUE partition function with even couplings

$$
Z_{N}(\mathbf{s})=\frac{(2 \pi)^{-N}}{\operatorname{Vol}(N)} \int_{\mathcal{H}(N)} e^{-N \operatorname{tr} V(M ; \mathbf{s})} d M
$$

Here, $V(M ; \mathbf{s})$ is an even polynomial of $M$

$$
V(M ; \mathbf{s})=\frac{1}{2} M^{2}-\sum_{j \geq 1} s_{j} M^{2 j},
$$

or, more generally, a power series, by $\mathbf{s}=\left(s_{1}, s_{2}, s_{3}, \ldots\right)$ we denote the collection of coefficients ${ }^{1}$ of $V(M)$, and by $\operatorname{Vol}(N)$ the volume of the quotient of the unitary group over the maximal torus $[U(1)]^{N}$

$$
\operatorname{Vol}(N)=\operatorname{Vol}\left(U(N) /[U(1)]^{N}\right)=\frac{\pi^{\frac{N(N-1)}{2}}}{G(N+1)}, \quad G(N+1)=\prod_{n=1}^{N-1} n !
$$

The integral will be considered as a formal saddle point expansion with respect to the small parameter $\epsilon$. Introduce the 't Hooft coupling parameter $x$ by

$$
x:=N \epsilon .
$$

Reexpanding the free energy $\mathcal{F}_{N}(\mathbf{s}):=\log Z_{N}(\mathbf{s})$ in powers of $\epsilon$ and replacing the Barnes $G$-function by its asymptotic expansion yields ${ }^{2}$

$$
\mathcal{F}(x, \mathbf{s} ; \epsilon):=\left.\mathcal{F}_{N}(\mathbf{s})\right|_{N=\frac{x}{\epsilon}}-\frac{1}{12} \log \epsilon=\sum_{g \geq 0} \epsilon^{2 g-2} \mathcal{F}_{g}(x, \mathbf{s}) .
$$

The GUE free energy $\mathcal{F}(x, \mathbf{s} ; \epsilon)$ can be represented $[21,22,1,23]$ in the form

$$
\begin{aligned}
\mathcal{F}(x, \mathbf{s} ; \epsilon)= & \frac{x^{2}}{2 \epsilon^{2}}\left(\log x-\frac{3}{2}\right)-\frac{1}{12} \log x+\zeta^{\prime}(-1)+\sum_{g \geq 2} \epsilon^{2 g-2} \frac{B_{2 g}}{4 g(g-1) x^{2 g-2}} \\
& +\sum_{g \geq 0} \epsilon^{2 g-2} \sum_{k \geq 0} \sum_{i_{1}, \ldots, i_{k} \geq 1} a_{g}\left(i_{1}, \ldots, i_{k}\right) s_{i_{1}} \ldots s_{i_{k}} x^{2-2 g-(k-|i|)}, \\
a_{g}\left(i_{1}, \ldots, i_{k}\right) & =\sum_{\Gamma} \frac{1}{\# \operatorname{Sym} \Gamma}
\end{aligned}
$$

where the last summation is taken over all connected oriented ribbon graphs $\Gamma$ of genus $g$ with $k$ vertices of valencies $2 i_{1}, \ldots, 2 i_{k}$, \# Sym $\Gamma$ is the order of the symmetry group of $\Gamma$, and $|i|:=i_{1}+\cdots+i_{k}$.

Our goal is to compare the expansions (1.1.3) and (1.2.5).

\footnotetext{
${ }^{1}$ The notation here is slightly different from that of $[7,8]$ where the coefficient of $M^{2 j}$ was denoted by $s_{2 j}$.

${ }^{2}$ It is often called $1 / N$-expansion as $\epsilon \sim 1 / N$.
} 


\subsection{From cubic Hodge integrals to random matrices. Main Conjecture.}

It was already observed by E. Witten [27] that the GUE partition function with an even polynomial $V(M)$ is tau-function of a particular solution to the Volterra (also called the discrete KdV) hierarchy. Recall that the first equation of the hierarchy (the Volterra lattice equation) reads

$$
\dot{w}_{n}=w_{n}\left(w_{n+1}-w_{n-1}\right)
$$

where

$$
w_{n}=\frac{Z_{n+1} Z_{n-1}}{Z_{n}^{2}}
$$

the time derivative is with respect to the variable $t=N s_{1}$. Other couplings $s_{k}$ are identified with the time variables of higher flows of the hierarchy. On another side, the study [12] of integrable systems associated with the Hodge integrals ${ }^{3}$ suggested the following conjectural statement: the Hodge partition function $Z_{\mathbb{E}}=e^{\mathcal{H}}$ of the form (1.1.3) as function of independent parameters $t_{i}$ is also a taufunction of the Volterra hierarchy. This observation provides a motivation for the main conjecture of the present paper.

It will be convenient to change normalisation of the GUE couplings. Put

$$
\bar{s}_{k}:=\left(\begin{array}{c}
2 k \\
k
\end{array}\right) s_{k}
$$

Conjecture 1.3.1 (Main Conjecture) The following formula holds true

$$
\begin{gathered}
\sum_{g=0}^{\infty} \epsilon^{2 g-2} \mathcal{F}_{g}(x, \mathbf{s})+\epsilon^{-2}\left(-\frac{1}{2} \sum_{k_{1}, k_{2} \geq 1} \frac{k_{1} k_{2}}{k_{1}+k_{2}} \bar{s}_{k_{1}} \bar{s}_{k_{2}}+\sum_{k \geq 1} \frac{k}{1+k} \bar{s}_{k}-x \sum_{k \geq 1} \bar{s}_{k}-\frac{1}{4}+x\right) \\
=\cosh \left(\frac{\epsilon \partial_{x}}{2}\right)\left[\sum_{g=0}^{\infty} \epsilon^{2 g-2} 2^{g} \mathcal{H}_{g}(\mathbf{t}(x, \mathbf{s}))\right] .
\end{gathered}
$$

where

$$
t_{i}(x, \mathbf{s}):=\sum_{k \geq 1} k^{i+1} \bar{s}_{k}-1+\delta_{i, 1}+x \cdot \delta_{i, 0}, \quad i \geq 0 .
$$

\subsection{Computational aspects of the Main Conjecture: how do we verify it?}

We will check validity of the Main Conjecture for small genera. Begin with $g=0$. Let us start with $\mathcal{H}_{0}(\mathbf{t})$. Instead of the explicit expansion (1.1.5) we use the following well known representation

$$
\mathcal{H}_{0}=\frac{v^{3}}{6}-\sum_{i \geq 0} t_{i} \frac{v^{i+2}}{i !(i+2)}+\frac{1}{2} \sum_{i, j \geq 0} t_{i} t_{j} \frac{v^{i+j+1}}{(i+j+1) i ! j !}
$$

where $v=v(\mathbf{t})=t_{0}+\ldots$ is the unique series solution to the equation

$$
v=\sum_{i \geq 0} t_{i} \frac{v^{i}}{i !} .
$$

\footnotetext{
${ }^{3}$ The first example of an integrable system associated with linear Hodge integrals was investigated by A. Buryak. In this case the integrable system was proved to be Miura equivalent to the Intermediate Long Wave equation [3].
} 
Here we recall that

$$
v=\frac{\partial^{2} \mathcal{H}_{0}(\mathbf{t})}{\partial t_{0}^{2}}=\sum_{k=1}^{\infty} \frac{1}{k} \sum_{i_{1}+\cdots+i_{k}=k-1} \frac{t_{i_{1}}}{i_{1} !} \ldots \frac{t_{i_{k}}}{i_{k} !}
$$

is a particular solution to the Riemann-Hopf hierarchy

$$
\frac{\partial v}{\partial t_{k}}=\frac{v^{k}}{k !} \frac{\partial v}{\partial t_{0}}, \quad k=0,1,2, \ldots
$$

For the genus zero GUE free energy $\mathcal{F}_{0}=\mathcal{F}_{0}(x, \mathbf{s})$ one has a similar representation. Like above, introduce

$$
u(x, \mathbf{s})=\frac{\partial^{2} \mathcal{F}_{0}(x, \mathbf{s})}{\partial x^{2}}
$$

and put

$$
w(x, \mathbf{s})=e^{u(x, \mathbf{s})}
$$

Proposition 1.4.1 The function $w=w(x, \mathbf{s})$ is the unique series solution to the equation

$$
w=x+\sum_{k \geq 1} k \bar{s}_{k} w^{k}, \quad \bar{s}_{k}:=\left(\begin{array}{c}
2 k \\
k
\end{array}\right) s_{k}, \quad w(x, \mathbf{s})=x+\ldots
$$

The genus zero GUE free energy $\mathcal{F}_{0}$ with even couplings has the following expression

$$
\mathcal{F}_{0}=\frac{w^{2}}{4}-x w+\sum_{k \geq 1} \bar{s}_{k}\left(x w^{k}-\frac{k}{k+1} w^{k+1}\right)+\frac{1}{2} \sum_{k_{1}, k_{2} \geq 1} \frac{k_{1} k_{2}}{k_{1}+k_{2}} \bar{s}_{k_{1}} \bar{s}_{k_{2}} w^{k_{1}+k_{2}}+\frac{x^{2}}{2} \log w .
$$

Clearly $w$ also satisfies the Riemann-Hopf hierarchy in a different normalization

$$
\frac{\partial w}{\partial \bar{s}_{k}}=k w^{k} \frac{\partial w}{\partial x}, \quad k \geq 1 .
$$

The solution can be written explicitly in the form essentially equivalent to (1.4.3)

$$
w=\sum_{n=1}^{\infty} \frac{1}{n} \sum_{i_{1}+\cdots+i_{n}=n-1} \operatorname{wt}\left(i_{1}\right) \ldots \operatorname{wt}\left(i_{n}\right) \bar{s}_{i_{1}} \ldots \bar{s}_{i_{n}}
$$

where we put $\bar{s}_{0}=x$ and denote

$$
\operatorname{wt}(i)= \begin{cases}1, & i=0 \\ i, & \text { otherwise. }\end{cases}
$$

It is now straightforward to verify that the substitution (1.3.2) yields

$$
e^{v(\mathbf{t}(x, \mathbf{s}))}=w(x, \mathbf{s}), \quad \text { i.e. } v(\mathbf{t}(x, \mathbf{s}))=u(x, \mathbf{s})
$$

and

$$
\mathcal{H}_{0}(\mathbf{t}(x, \mathbf{s}))=\mathcal{F}_{0}(x, \mathbf{s})-\frac{1}{2} \sum_{k_{1}, k_{2} \geq 1} \frac{k_{1} k_{2}}{k_{1}+k_{2}} \bar{s}_{k_{1}} \bar{s}_{k_{2}}+\sum_{k \geq 1} \frac{k}{1+k} \bar{s}_{k}-x \sum_{k \geq 1} \bar{s}_{k}-\frac{1}{4}+x .
$$


See in Sect. 2 for the details of this computation.

In order to proceed to higher genera we will use the method that goes back to the paper [5] by R. Dijkgraaf and E. Witten. The idea of this method is to express the positive genus free energy terms via the genus zero. Let us first explain this method for the Hodge free energy.

Theorem 1.4.2 ([12]) There exist functions $H_{g}\left(v, v_{1}, v_{2}, \ldots, v_{3 g-2}\right), g \geq 1$ of independent variables $v, v_{1}, v_{2}, \ldots$ such that

$$
\mathcal{H}_{g}(\mathbf{t})=H_{g}\left(v(\mathbf{t}), \frac{\partial v(\mathbf{t})}{\partial t_{0}}, \ldots, \frac{\partial^{3 g-2} v(\mathbf{t})}{\partial t_{0}^{3 g-2}}\right), \quad g \geq 1 .
$$

Here $v(\mathbf{t})$ is given by eq. (1.4.3). Moreover, for any $g \geq 2$ the function $H_{g}$ is a polynomial in the variables $v_{2}, \ldots, v_{3 g-2}$ with coefficients in $\mathbb{Q}\left[v_{1}, v_{1}^{-1}\right]$ (independent of $v$ ).

Explicitly,

$$
\begin{gathered}
H_{1}\left(v, v_{1}\right)=-\frac{1}{16} v+\frac{1}{24} \log v_{1} \\
H_{2}\left(v_{1}, v_{2}, v_{3}, v_{4}\right)=\frac{7 v_{2}}{2560}-\frac{v_{1}^{2}}{11520}+\frac{v_{4}}{1152 v_{1}^{2}}-\frac{v_{3}}{320 v_{1}}+\frac{v_{2}^{3}}{360 v_{1}^{4}}+\frac{11 v_{2}^{2}}{3840 v_{1}^{2}}-\frac{7 v_{3} v_{2}}{1920 v_{1}^{3}},
\end{gathered}
$$

etc. The algorithm for computing the functions $H_{g}$ can be found in [12]. They were used in the construction of the associated integrable hierarchy via the quasi-triviality transformation approach $[10]$.

Let us now proceed to the higher genus terms for the random matrix free energy (recall that only even couplings are allowed).

Theorem 1.4.3 There exist functions $F_{g}\left(v, v_{1}, \ldots, v_{3 g-2}\right), g \geq 1$ of independent variables $v, v_{1}, v_{2}$, ... such that

$$
\mathcal{F}_{g}(x, \mathbf{s})=F_{g}\left(u(x, \mathbf{s}), \frac{\partial u(x, \mathbf{s})}{\partial x}, \ldots, \frac{\partial^{3 g-2} u(x, \mathbf{s})}{\partial x^{3 g-2}}\right), \quad g \geq 1 .
$$

Here

$$
u(x, \mathbf{s})=\frac{\partial^{2} \mathcal{F}_{0}(x, \mathbf{s})}{\partial x^{2}}=\log w(x, \mathbf{s}) .
$$

Recall that the function $w(x, \mathbf{s})$ is determined from eq. (1.4.6).

Explicitly

$$
F_{1}\left(v, v_{1}\right)=\frac{1}{12} \log v_{1}+\text { const }
$$

with const $=\frac{i \pi}{24}+\zeta^{\prime}(-1)$,

$$
F_{2}\left(v_{1}, v_{2}, v_{3}, v_{4}\right)=-\frac{v_{2}}{480}-\frac{v_{1}^{2}}{2880}+\frac{v_{4}}{288 v_{1}^{2}}-\frac{v_{3}}{480 v_{1}}+\frac{v_{2}^{3}}{90 v_{1}^{4}}+\frac{v_{2}^{2}}{960 v_{1}^{2}}-\frac{7 v_{3} v_{2}}{480 v_{1}^{3}}
$$

etc. For any $g \geq 2$ the function $F_{g}$ is a polynomial in the variables $v_{2}, \ldots, v_{3 g-2}$ with coefficients in $\mathbb{Q}\left[v_{1}, v_{1}^{-1}\right]$. 
Using the fact that $\partial_{t_{0}}=\partial_{x}$ (see Section 3.2 below) along with the standard expansion

$$
\cosh \left(\frac{\epsilon \partial_{x}}{2}\right)=1+\sum_{n \geq 1} \frac{1}{(2 n) !}\left(\frac{\epsilon}{2}\right)^{2 n} \partial_{x}^{2 n}
$$

we recast the Main Conjecture for $g \geq 1$ into a sequence of the following relationships between the functions $F_{g}$ and $H_{g}$

$$
F_{1}=2 H_{1}+\frac{v}{8}+\text { const }
$$

and, for $g \geq 2$

$$
F_{g}\left(v_{1}, \ldots, v_{3 g-2}\right)=\frac{v_{2 g-2}}{2^{2 g}(2 g) !}+\frac{D_{0}^{2 g-2} H_{1}\left(v ; v_{1}\right)}{2^{2 g-3}(2 g-2) !}+\sum_{m=2}^{g} \frac{2^{3 m-2 g}}{(2 g-2 m) !} D_{0}^{2(g-m)} H_{m}\left(v_{1}, \ldots, v_{3 m-2}\right)
$$

where the operator $D_{0}$ is defined by

$$
D_{0}=v_{1} \frac{\partial}{\partial v}+\sum_{k \geq 1} v_{k+1} \frac{\partial}{\partial v_{k}}
$$

For example,

$$
F_{2}\left(v_{1}, v_{2}, v_{3}, v_{4}\right)=4 H_{2}\left(v_{1}, v_{2}, v_{3}, v_{4}\right)+\frac{1}{4} D_{0}^{2} H_{1}+\frac{1}{384} v_{2}
$$

Eqs. (1.4.16), (1.4.18) can be easily verified (see below). In order to verify validity of eqs. (1.4.17) for any $g \geq 2$ we write a conjectural explicit expression for the functions $F_{g}\left(v_{1}, \ldots, v_{3 g-2}\right)$ responsible for the genus $g$ random matrix free energies. This will be done in the next subsection.

\subsection{An explicit expression for $F_{g}$}

We first recall some notations. $\mathbb{Y}$ will denote the set of all partitions. For any partition $\lambda \in \mathbb{Y}$ denote by $\ell(\lambda)$ the length of $\lambda$, by $\lambda_{1}, \lambda_{2}, \ldots, \lambda_{\ell(\lambda)}$ the non-zero components, $|\lambda|=\lambda_{1}+\cdots+\lambda_{\ell(\lambda)}$ the weight, and by $m_{i}(\lambda)$ the multiplicity of $i$ in $\lambda$. Put $m(\lambda) !:=\prod_{i \geq 1} m_{i}(\lambda)$ !. The set of all partitions of weight $k$ will be denoted by $\mathbb{Y}_{k}$. For an arbitrary sequence of variables $v_{1}, v_{2}, \ldots$, denote $v_{\lambda}=v_{\lambda_{1}} \cdots v_{\lambda_{\ell(\lambda)}}$.

Conjecture 1.5.1 For any $g \geq 2$, the genus $g$ GUE free energy $F_{g}$ has the following expression

$$
\begin{gathered}
F_{g}\left(v_{1}, \ldots, v_{3 g-2}\right)=\frac{v_{2 g-2}}{2^{2 g}(2 g) !}+\frac{1}{2^{2 g-3}(2 g-2) !} D_{0}^{2 g-2}\left(-\frac{1}{16} v+\frac{1}{24} \log v_{1}\right)+\sum_{m=2}^{g} \frac{2^{3 m-2 g}}{(2 g-2 m) !} \\
\sum_{k=0}^{3 m-3} \sum_{\substack{k_{1}+k_{2}+k_{3}=k \\
0 \leq k_{1}, k_{2}, k_{3} \leq m}} \frac{(-1)^{k_{2}+k_{3}}}{2^{k_{1}}} \sum_{\rho, \mu \in \mathbb{Y}_{3 m-3-k}} \frac{\left\langle\lambda_{k_{1}} \lambda_{k_{2}} \lambda_{k_{3}} \tau_{\rho+1}\right\rangle_{g}}{m(\rho) !} Q^{\rho \mu} D_{0}^{2 g-2 m}\left(\frac{v_{\mu+1}}{v_{1}^{\ell(\mu)+m-1-k}}\right)
\end{gathered}
$$

where for a partition $\mu=\left(\mu_{1}, \ldots, \mu_{\ell}\right), \mu+1$ denotes the partition $\left(\mu_{1}+1, \ldots, \mu_{\ell}+1\right), Q^{\rho \mu}$ is the so-called Q-matrix defined by

$$
Q^{\rho \mu}=(-1)^{\ell(\rho)} \sum_{\substack{\mu^{1} \in \mathbb{Y}_{\lambda_{1}}, \ldots, \mu^{\ell(\rho)} \in \mathbb{Y}_{\lambda_{\ell(\rho)}} \\ \cup_{q=1}^{\ell(\rho)} \mu^{q}=\mu}} \prod_{\substack{q=1 \\(\rho)}} \frac{\left(\rho_{q}+\ell\left(\mu^{q}\right)\right) !(-1)^{\ell\left(\mu^{q}\right)}}{m\left(\mu^{q}\right) ! \prod_{j=1}^{\infty}(j+1) !^{m_{j}\left(\mu^{q}\right)}} .
$$


In this formula we have used the notation

$$
\left\langle\lambda_{k_{1}} \lambda_{k_{2}} \lambda_{k_{3}} \tau_{\nu}\right\rangle_{g}:=\int_{\overline{\mathcal{M}}_{g, \ell}} \lambda_{k_{1}} \lambda_{k_{2}} \lambda_{k_{3}} \psi_{1}^{\nu_{1}} \ldots \psi_{\ell}^{\nu_{\ell}}, \quad \forall \nu=\left(\nu_{1}, \ldots, \nu_{\ell}\right) \in \mathbb{Y} .
$$

Details about $Q$-matrix can be found in [9]. Conj.1.5.1 indicates that the the special cubic Hodge integrals (1.1.2) naturally appear in the expressions for the higher genus terms of GUE free energy.

Organization of the paper In Sect. 2 we review the approach of $[10,7]$ to the GUE free energy, and prove Prop.1.4.1 and Thm.1.4.3. In Sect. 3 we verify Conj.1.3.1 and Conj.1.5.1 up to the genus 2 approximation, and give explicit formulae of $\mathcal{F}_{g}$ for $g=3,4,5$.

Acknowledgements We wish to thank Si-Qi Liu and Youjin Zhang for helpful discussions.

\section{GUE free energy with even valencies}

\subsection{Calculating the GUE free energy from Frobenius manifold of $\mathbb{P}^{1}$ topological $\sigma$-model}

It is known that the GUE partition function $Z_{N}$ (with even and odd couplings) is the tau-function of a particular solution to the Toda lattice hierarchy. Using this fact, one of the authors in [7] developed an efficient algorithm of calculating of GUE free energy, which is an application of the general approach of $[10,6]$ for the particular example of the two-dimensional Frobenius manifold with potential

$$
F=\frac{1}{2} u v^{2}+e^{u}
$$

(Warning: only in this section, the notation $v$ is different from that of the Introduction.) In this section, we give a brief reminder of this approach referring the readers to $[10,11,7]$ for details.

Introduce two analytic functions $\theta_{1}(u, v ; z), \theta_{2}(u, v ; z)$ as follows

$$
\begin{aligned}
& \theta_{1}(u, v ; z)=-2 e^{z v} \sum_{m=0}^{\infty}\left(-\frac{1}{2} u+c_{m}\right) e^{m u} \frac{z^{2 m}}{m !^{2}}=: \sum_{p \geq 0} \theta_{1, p}(u, v) z^{p} \\
& \theta_{2}(u, v ; z)=z^{-1}\left(\sum_{m \geq 0} e^{m u+z v} \frac{z^{2 m}}{(m !)^{2}}-1\right)=: \sum_{p \geq 0} \theta_{2, p}(u, v) z^{p} .
\end{aligned}
$$

Here $c_{m}=\sum_{k=1}^{m} \frac{1}{k}$ denotes the $m$-th harmonic number.

Note that, as in the Introduction, we will only consider the GUE partition function with even couplings. The corresponding Euler-Lagrange equation $[7,6,10]$ reads

$$
\begin{aligned}
& x-w+\sum_{k \geq 1}(2 k) ! s_{k} \sum_{m=1}^{k} m w^{m} \frac{v^{2 k-2 m}}{(2 k-2 m) ! m !^{2}}=0 \\
& -v+\sum_{k \geq 1}(2 k) ! s_{k} \sum_{m=0}^{k-1} w^{m} \frac{v^{2 k-1-2 m}}{(2 k-1-2 m) ! m !^{2}}=0
\end{aligned}
$$


where $w=e^{u}$ (as in the Introduction). Note that we are only interested in the unique series solution $(v(x, \mathbf{s}), w(x, \mathbf{s}))$ of $(2.1 .3),(2.1 .4)$ such that $v(x, \mathbf{0})=0, w(x, \mathbf{0})=x$. It is then easy to see from eq. (2.1.4) that

$$
v=v(x, \mathbf{s}) \equiv 0
$$

And eq. (2.1.3) becomes

$$
x-w+\sum_{m \geq 1} s_{2 m} m w^{m} \frac{(2 m) !}{m !^{2}}=0 .
$$

Define a family of analytic functions $\Omega_{\alpha, p ; \beta, q}(u, v)$ by the following generating formula

$$
\sum_{p, q \geq 0} \Omega_{\alpha, p ; \beta, q} z^{p} y^{q}=\frac{1}{z+y}\left[\frac{\partial \theta_{\alpha}(z)}{\partial v} \frac{\partial \theta_{\beta}(y)}{\partial u}+\frac{\partial \theta_{\alpha}(z)}{\partial u} \frac{\partial \theta_{\beta}(y)}{\partial v}-\delta_{\alpha+\beta, 3}\right], \quad \alpha, \beta=1,2 .
$$

The genus zero GUE free energy $\mathcal{F}_{0}(x, \mathbf{s})$ then has the following expression

$$
\begin{aligned}
\mathcal{F}_{0}= & \frac{1}{2} \sum_{p, q \geq 2}(2 p) !(2 q) ! s_{p} s_{q} \Omega_{2,2 p-1 ; 2,2 q-1}+x \sum_{q \geq 1}(2 q) ! s_{q} \Omega_{1,0 ; 2,2 q-1}-x \Omega_{1,0 ; 2,1} \\
& +\frac{1}{2}\left(1-2 s_{1}\right)^{2} \Omega_{2,1 ; 2,1}+\sum_{q \geq 2}\left(2 s_{1}-1\right)(2 q) ! s_{q} \Omega_{2,1 ; 2,2 q-1}+\frac{1}{2} x^{2} \Omega_{1,0 ; 1,0} .
\end{aligned}
$$

The higher genus terms in the $1 / N$ expansion of the GUE free energy can be determined recursively from the loop equation $[10,7]$ for a sequence of functions

$$
F_{g}=F_{g}\left(u, v, u_{1}, v_{1}, \ldots, v_{3 g-2}, u_{3 g-2}\right), \quad g \geq 1 .
$$

This equation has the following form

$$
\begin{aligned}
& \sum_{r \geq 0}\left[\frac{\partial \Delta \mathcal{F}}{\partial v_{r}}\left(\frac{v-\lambda}{D}\right)_{r}-2 \frac{\partial \Delta \mathcal{F}}{\partial u_{r}}\left(\frac{1}{D}\right)_{r}\right] \\
& +\sum_{r \geq 1} \sum_{k=1}^{r}\left(\begin{array}{c}
r \\
k
\end{array}\right)\left(\frac{1}{\sqrt{D}}\right)_{k-1}\left[\frac{\partial \Delta \mathcal{F}}{\partial v_{r}}\left(\frac{v-\lambda}{\sqrt{D}}\right)_{r-k+1}-2 \frac{\partial \Delta \mathcal{F}}{\partial u_{r}}\left(\frac{1}{\sqrt{D}}\right)_{r-k+1}\right] \\
= & D^{-3} e^{u}\left(4 e^{u}+(v-\lambda)^{2}\right)-\epsilon^{2} \sum_{k, l}\left[\frac{1}{4} S\left(\Delta \mathcal{F}, v_{k}, v_{l}\right)\left(\frac{v-\lambda}{\sqrt{D}}\right)_{k+1}\left(\frac{v-\lambda}{\sqrt{D}}\right)_{l+1}\right. \\
& \left.-S\left(\Delta \mathcal{F}, v_{k}, u_{l}\right)\left(\frac{v-\lambda}{\sqrt{D}}\right)_{k+1}\left(\frac{1}{\sqrt{D}}\right)_{l+1}+S\left(\Delta \mathcal{F}, u_{k}, u_{l}\right)\left(\frac{1}{\sqrt{D}}\right)_{k+1}\left(\frac{1}{\sqrt{D}}\right)_{l+1}\right] \\
& -\frac{\epsilon^{2}}{2} \sum_{k}\left[\frac{\partial \Delta \mathcal{F}}{\partial v_{k}} \frac{4 e^{u}(v-\lambda) u_{1}-T v_{1}}{D^{3}}+\frac{\partial \Delta \mathcal{F}}{\partial u_{k}} \frac{4(v-\lambda) v_{1}-T u_{1}}{D^{3}}\right]\left(e^{u}\right)_{k+1}
\end{aligned}
$$

where $\triangle \mathcal{F}=\sum_{g \geq 1} \epsilon^{2 g} F_{g}, D=(v-\lambda)^{2}-4 e^{u}, T=(v-\lambda)^{2}+4 e^{u}, S(f, a, b):=\frac{\partial^{2} f}{\partial a \partial b}+\frac{\partial f}{\partial a} \frac{\partial f}{\partial b}$, and $f_{r}$ stands for $\partial_{x}^{r}(\bar{f})$. Solution $\Delta \mathcal{F}$ of (2.1.8) exists and is unique up to an additive constant. $F_{g}$ is a polynomial in $u_{2}, v_{2}, \ldots, u_{3 g-2}, v_{3 g-2}$. For $g \geq 2, F_{g}$ is a rational function of $u_{1}, v_{1}$. Then [10] the genus $g$ term in the expansion (1.2.4), in the particular case of even couplings only, reads

$$
\mathcal{F}_{g}(x, \mathbf{s})=F_{g}\left(u(x, \mathbf{s}), v=0, \frac{\partial u(x, \mathbf{s})}{\partial x}, v_{x}=0, \ldots, \frac{\partial^{3 g-2} u(x, \mathbf{s})}{\partial x^{3 g-2}}, v_{3 g-2}=0\right), \quad g \geq 1
$$

This procedure will be used in the next subsection. 


\subsection{Proof of Prop. 1.4.1, Thm. 1.4.3}

Proof of Prop. 1.4.1. Noting that

$$
\begin{aligned}
& \theta_{2}(u, 0, z)=z^{-1}\left(\sum_{m \geq 0} w^{m} \frac{z^{2 m}}{(m !)^{2}}-1\right), \\
& \partial_{v} \theta_{2}(u, 0, z)=\sum_{m \geq 0} w^{m} \frac{z^{2 m}}{(m !)^{2}}, \quad \partial_{u} \theta_{2}(u, 0, z)=\sum_{m \geq 0} m w^{m} \frac{z^{2 m-1}}{(m !)^{2}}
\end{aligned}
$$

and using (2.1.6) we have

$$
\sum_{p, q \geq 0} \Omega_{2, p ; 2, q} z^{p} y^{q}=\frac{\sum_{m \geq 0} w^{m} \frac{z^{2 m}}{(m !)^{2}} \sum_{m \geq 0} m w^{m} \frac{y^{2 m-1}}{(m !)^{2}}+\sum_{m \geq 0} m w^{m} \frac{z^{2 m-1}}{(m !)^{2}} \sum_{m \geq 0} w^{m} \frac{y^{2 m}}{(m !)^{2}}}{z+y} .
$$

It follows that if $p+q$ is odd then $\Omega_{2, p ; 2, q}$ vanishes; otherwise, we have

$$
\begin{aligned}
\Omega_{2, p ; 2, q} & =\frac{w^{\frac{p+q}{2}+1}}{\left(1+\frac{p+q}{2}\right)\left[\left(\frac{p}{2}\right) !\right]^{2}\left[\left(\frac{q}{2}\right) !\right]^{2}}, \quad p, q \text { are both even; } \\
\Omega_{2, p ; 2, q} & =\frac{\frac{p+1}{2} \frac{q+1}{2} w^{\frac{p+q}{2}+1}}{\left(1+\frac{p+q}{2}\right)\left[\left(\frac{p+1}{2}\right) !\right]^{2}\left[\left(\frac{q+1}{2}\right) !\right]^{2}}, \quad p, q \text { are both odd. }
\end{aligned}
$$

Substituting these expressions in (2.1.7) we obtain

$$
\begin{aligned}
& \mathcal{F}_{0}=\frac{1}{2} x^{2} u+\frac{1}{2} \sum_{k_{1}, k_{2} \geq 0}\left(2 k_{1}+2\right) !\left(2 k_{2}+2\right) ! s_{k_{1}+1} s_{k_{2}+1} \frac{\left(k_{1}+1\right)\left(k_{2}+1\right) w^{k_{1}+k_{2}+2}}{\left(k_{1}+k_{2}+2\right)\left[\left(k_{1}+1\right) !\right]^{2}\left[\left(k_{2}+1\right) !\right]^{2}} \\
& +x \sum_{k \geq 0}(2 k+2) ! s_{k+1} \frac{w^{k+1}}{(k+1) !^{2}}-x w+\left(1-4 s_{1}\right) \frac{w^{2}}{4}-\sum_{k \geq 1}(2 k+2) ! s_{k+1} \frac{(k+1) w^{k+2}}{(k+2)[(k+1) !]^{2}} .
\end{aligned}
$$

Equation (1.4.6) is already proved in (2.1.5). The proposition is proved.

Proof of Theorem 1.4.3. For $g=1,2$, taking $v=v_{1}=v_{2}=\cdots=0$ in the general expressions of $F_{g}\left(u, v, u_{1}, v_{1}, \ldots, u_{3 g-2}, v_{3 g-2}\right)[10,11]$ one obtains (1.4.14) and (1.4.15). For any $g \geq 1$, the existence of $F_{g}\left(u, u_{1}, \ldots, u_{3 g-2}\right)$ such that

$$
\mathcal{F}_{g}(x, \mathbf{s})=F_{g}\left(u(x, \mathbf{s}), \frac{\partial u(x, \mathbf{s})}{\partial x}, \ldots, \frac{\partial^{3 g-2} u(x, \mathbf{s})}{\partial x^{3 g-2}}\right)
$$

is a direct result of $[10,11]$ when taking $v=v_{1}=v_{2}=\cdots=0$ in $F_{g}\left(u, v, u_{1}, v_{1}, \ldots, u_{3 g-2}, v_{3 g-2}\right)$.

\section{Verification of the Main Conjecture for low genera}

\subsection{Genus 0}

Recall that the genus zero cubic Hodge free energy can be expressed as

$$
\mathcal{H}_{0}(\mathbf{t})=\frac{1}{2} \sum_{i, j \geq 0} \tilde{t}_{i} \tilde{t}_{j} \Omega_{i ; j}(v(\mathbf{t})) .
$$


where $\tilde{t}_{i}=t_{i}-\delta_{i, 1}, \mathbf{t}=\left(t_{0}, t_{1}, t_{2}, \ldots\right), \Omega_{i ; j}$ are polynomials in $v$ given by

$$
\Omega_{i ; j}(v)=\frac{v^{i+j+1}}{(i+j+1) i ! j !}
$$

and $v(\mathbf{t})$ is the unique series solution to the following Euler-Lagrange equation of the one-dimensional Frobenius manifold

$$
v=\sum_{i \geq 0} t_{i} \frac{v^{i}}{i !} .
$$

(Warning: the above $v$ is the flat coordinate of the one-dimensional Frobenius manifold; avoid confusing with $v$ in Section 2 where $(u, v)$ are flat coordinates of the two-dimensional Frobenius manifold of $\mathbb{P}^{1}$ topological $\sigma$-model.)

Let us consider the following substitution of time variables

$$
t_{i}=\sum_{k \geq 1} k^{i+1} \bar{s}_{k}-1+\delta_{i, 1}+x \cdot \delta_{i, 0}, \quad i \geq 0 .
$$

Note that with this substitution the cubic Hodge free energies will be considered to be expanded at $x=1$. We have $\tilde{t}_{i}=\sum_{k \geq 1} k^{i+1} \bar{s}_{k}-1+x \cdot \delta_{i, 0}$, and so

$$
\begin{aligned}
\mathcal{H}_{0}= & \frac{1}{2} \sum_{i, j \geq 0} \tilde{t}_{i} \tilde{t}_{j} \Omega_{i ; j}(v(\mathbf{t})) \\
= & \frac{1}{2} \sum_{i, j \geq 0}\left(\sum_{k_{1} \geq 1} k_{1}^{i+1} \bar{s}_{k_{1}}-1+x \cdot \delta_{i, 0}\right)\left(\sum_{k_{2} \geq 1} k_{2}^{j+1} \bar{s}_{k_{2}}-1+x \cdot \delta_{j, 0}\right) \frac{v^{i+j+1}}{(i+j+1) i ! j !} \\
= & \frac{1}{2} \sum_{i, j \geq 0} \sum_{k_{1}, k_{2} \geq 1} k_{1}^{i+1} k_{2}^{j+1} \bar{s}_{k_{1}} \bar{s}_{k_{2}} \frac{v^{i+j+1}}{(i+j+1) i ! j !}-\sum_{i, j \geq 0} \sum_{k_{1} \geq 1} k_{1}^{i+1} \bar{s}_{k_{1}} \frac{v^{i+j+1}}{(i+j+1) i ! j !} \\
& +x \sum_{i, j \geq 0} \sum_{k_{1} \geq 1} k_{1}^{i+1} \bar{s}_{k_{1}} \delta_{j, 0} \frac{v^{i+j+1}}{(i+j+1) i ! j !}+\frac{1}{2} \sum_{i, j \geq 0} \frac{v^{i+j+1}}{(i+j+1) i ! j !} \\
& -x \sum_{i, j \geq 0} \delta_{j, 0} \frac{v^{i+j+1}}{(i+j+1) i ! j !}+\frac{x^{2}}{2} \sum_{i, j \geq 0} \delta_{i, 0} \delta_{j, 0} \frac{v^{i+j+1}}{(i+j+1) i ! j !} .
\end{aligned}
$$

We simplify it term by term:

$$
\begin{aligned}
& \frac{x^{2}}{2} \sum_{i, j \geq 0} \delta_{i, 0} \delta_{j, 0} \frac{v^{i+j+1}}{(i+j+1) i ! j !}=\frac{x^{2}}{2} v, \\
& x \sum_{i, j \geq 0} \delta_{j, 0} \frac{v^{i+j+1}}{(i+j+1) i ! j !}=x\left(e^{v}-1\right), \\
& \frac{1}{2} \sum_{i, j \geq 0} \frac{v^{i+j+1}}{(i+j+1) i ! j !}=\frac{1}{2} \sum_{\ell \geq 0} \sum_{i=0}^{\ell} \frac{v^{\ell+1} \ell !}{(\ell+1) ! i !(\ell-i) !}=\frac{1}{2} \sum_{\ell \geq 0} \frac{v^{\ell+1} 2^{\ell}}{(\ell+1) !}=\frac{1}{4}\left(e^{2 v}-1\right), \\
& x \sum_{i, j \geq 0} \sum_{k_{1} \geq 1} k_{1}^{i+1} \bar{s}_{k_{1}} \delta_{j, 0} \frac{v^{i+j+1}}{(i+j+1) i ! j !}=x \sum_{i \geq 0} \sum_{k_{1} \geq 1} k_{1}^{i+1} \bar{s}_{k_{1}} \frac{v^{i+1}}{(i+1) !}=x \sum_{k \geq 1} \bar{s}_{k}\left(e^{k v}-1\right),
\end{aligned}
$$




$$
\begin{gathered}
\sum_{i, j \geq 0} \sum_{k_{1} \geq 1} k_{1}^{i+1} \bar{s}_{k_{1}} \frac{v^{i+j+1}}{(i+j+1) i ! j !}=\sum_{k \geq 1} k \bar{s}_{k} \sum_{\ell \geq 0}(1+k)^{\ell} \frac{v^{\ell+1}}{(\ell+1) !}=\sum_{k \geq 1} \frac{k}{1+k} \bar{s}_{k}\left(e^{(1+k) v}-1\right), \\
\frac{1}{2} \sum_{i, j \geq 0} \sum_{k_{1}, k_{2} \geq 1} k_{1}^{i+1} k_{2}^{j+1} \bar{s}_{k_{1}} \bar{s}_{k_{2}} \frac{v^{i+j+1}}{(i+j+1) i ! j !}=\frac{1}{2} \sum_{k_{1}, k_{2} \geq 1} k_{1} k_{2} \bar{s}_{k_{1}} \bar{s}_{k_{2}} \sum_{\ell \geq 0} \frac{v^{\ell+1}}{(\ell+1) !}\left(k_{1}+k_{2}\right)^{\ell} \\
=\frac{1}{2} \sum_{k_{1}, k_{2} \geq 1} \frac{k_{1} k_{2}}{k_{1}+k_{2}} \bar{s}_{k_{1}} \bar{s}_{k_{2}}\left(e^{\left(k_{1}+k_{2}\right) v}-1\right) .
\end{gathered}
$$

Let $w=e^{v}$. We have

$$
\begin{aligned}
\mathcal{H}_{0}= & \frac{1}{2} \sum_{k_{1}, k_{2} \geq 1} \frac{k_{1} k_{2}}{k_{1}+k_{2}} \bar{s}_{k_{1}} \bar{s}_{k_{2}}\left(w^{k_{1}+k_{2}}-1\right)-\sum_{k \geq 1} \frac{k}{1+k} \bar{s}_{k}\left(w^{1+k}-1\right)+x \sum_{k \geq 1} \bar{s}_{k}\left(w^{k}-1\right) \\
& +\frac{1}{4}\left(w^{2}-1\right)-x(w-1)+\frac{x^{2}}{2} \log w .
\end{aligned}
$$

On the other hand, recall from Prop. 1.4.1 that the genus zero GUE free energy with even couplings has the form

$$
\mathcal{F}_{0}=\frac{w^{2}}{4}-x w+\sum_{k \geq 1} \bar{s}_{k}\left(x w^{k}-\frac{k}{k+1} w^{k+1}\right)+\frac{1}{2} \sum_{k_{1}, k_{2} \geq 1} \frac{k_{1} k_{2}}{k_{1}+k_{2}} \bar{s}_{k_{1}} \bar{s}_{k_{2}} w^{k_{1}+k_{2}}+\frac{x^{2}}{2} \log w .
$$

Here $w$ is the power series solution to

$$
w=x+\sum_{k \geq 1} k \bar{s}_{k} w^{k}
$$

Recall that $w=e^{u}$; so

$$
e^{u}=x+\sum_{k \geq 1} k \bar{s}_{k} e^{k u}
$$

Namely,

$$
1+\sum_{j \geq 1} \frac{u^{j}}{j !}=x+\sum_{k \geq 1} k \bar{s}_{k}\left(1+\sum_{j \geq 1} \frac{k^{j} u^{j}}{j !}\right)
$$

It follows that

$$
u(x, \mathbf{s})=v(\mathbf{t}(x, \mathbf{s}))
$$

We conclude that

$$
\mathcal{H}_{0}(\mathbf{t}(x, \mathbf{s}))-\mathcal{F}_{0}(x, \mathbf{s})=-\frac{1}{2} \sum_{k_{1}, k_{2} \geq 1} \frac{k_{1} k_{2}}{k_{1}+k_{2}} \bar{s}_{k_{1}} \bar{s}_{k_{2}}+\sum_{k \geq 1} \frac{k}{1+k} \bar{s}_{k}-x \sum_{k \geq 1} \bar{s}_{k}-\frac{1}{4}+x .
$$

This finishes the proof of the genus zero part of the Main Conjecture. 


\subsection{Genus 1,2}

Note that the substitution (1.3.2)

$$
\left(t_{0}, t_{1}, t_{2}, \ldots\right) \mapsto\left(x, \bar{s}_{1}, \bar{s}_{2}, \ldots\right)
$$

satisfies that

$$
\begin{aligned}
\frac{\partial}{\partial x} & =\frac{\partial}{\partial t_{0}} \\
\frac{\partial}{\partial \bar{s}_{k}} & =\sum_{i \geq 0} k^{i+1} \frac{\partial}{\partial t_{i}}, \quad k \geq 1 .
\end{aligned}
$$

In particular, we have

$$
\frac{\partial v}{\partial t_{0}}(\mathbf{t}(x, \mathbf{s}))=\frac{\partial v(\mathbf{t}(x, \mathbf{s}))}{\partial x}=\frac{\partial u(x, \mathbf{s})}{\partial x} .
$$

The last equality is due to (3.1.2).

Recall, from the algorithm of [12], that the genus 1 special cubic Hodge free energy is given by

$$
H_{1}\left(v ; v_{1}\right)=\frac{1}{24} \log v_{1}-\frac{v}{16} .
$$

So

$$
2 H_{1}\left(v ; v_{1}\right)+\frac{v}{8}+\frac{i \pi}{24}+\zeta^{\prime}(-1)=\frac{1}{12} \log v_{1}+\frac{i \pi}{24}+\zeta^{\prime}(-1) .
$$

This proves the genus 1 part of the Main Conjecture.

The genus 2 term of the special cubic Hodge free energy is given by

$$
H_{2}\left(v_{1}, v_{2}, v_{3}, v_{4}\right)=\frac{7 v_{2}}{2560}-\frac{v_{1}^{2}}{11520}+\frac{v_{4}}{1152 v_{1}^{2}}-\frac{v_{3}}{320 v_{1}}+\frac{v_{2}^{3}}{360 v_{1}^{4}}+\frac{11 v_{2}^{2}}{3840 v_{1}^{2}}-\frac{7 v_{3} v_{2}}{1920 v_{1}^{3}} .
$$

So

$$
\begin{aligned}
4 H_{2}+\frac{1}{4} D_{0}^{2} H_{1}+\frac{1}{384} v_{2} & =4 H_{2}\left(v_{1}, v_{2}, v_{3}, v_{4}\right)-\frac{5}{384} v_{2}+\frac{1}{96}\left[\frac{v_{3}}{v_{1}}-\left(\frac{v_{2}}{v_{1}}\right)^{2}\right] \\
& =-\frac{v_{2}}{480}-\frac{v_{1}^{2}}{2880}+\frac{v_{4}}{288 v_{1}^{2}}-\frac{v_{3}}{480 v_{1}}+\frac{v_{2}^{3}}{90 v_{1}^{4}}+\frac{v_{2}^{2}}{960 v_{1}^{2}}-\frac{7 v_{3} v_{2}}{480 v_{1}^{3}} \\
& =F_{2}\left(v_{1}, v_{2}, v_{3}, v_{4}\right) .
\end{aligned}
$$

This proves the genus 2 part of the Main Conjecture.

\subsection{Genus 3,4}

Using the Main Conjecture along with the algorithm of [12], we obtain the following two statements. 
Conjecture 3.3.1 The genus 3 GUE free energy is given by

$$
\begin{aligned}
& F_{3}\left(u_{1}, \ldots, u_{7}\right) \\
= & \frac{13 u_{4}}{120960}+\frac{u_{2}^{2}}{24192}-\frac{u_{1}^{4}}{725760}+\frac{u_{7}}{10368 u_{1}^{3}}-\frac{u_{6}}{5760 u_{1}^{2}}-\frac{u_{5}}{13440 u_{1}} \\
& -\frac{103 u_{4}^{2}}{60480 u_{1}^{4}}+\frac{59 u_{3}^{3}}{8064 u_{1}^{5}}+\frac{u_{3}^{2}}{2688 u_{1}^{2}}+\frac{u_{3} u_{1}}{12096}-\frac{5 u_{2}^{6}}{81 u_{1}^{8}}-\frac{13 u_{2}^{5}}{1890 u_{1}^{6}} \\
& +\frac{5 u_{2}^{4}}{5376 u_{1}^{4}}-\frac{u_{2}^{3}}{9072 u_{1}^{2}}-\frac{7 u_{6} u_{2}}{5760 u_{1}^{4}}-\frac{53 u_{3} u_{5}}{20160 u_{1}^{4}}+\frac{353 u_{5} u_{2}^{2}}{40320 u_{1}^{5}}+\frac{u_{5} u_{2}}{840 u_{1}^{3}} \\
& +\frac{89 u_{3} u_{4}}{40320 u_{1}^{3}}-\frac{83 u_{4} u_{2}^{3}}{1890 u_{1}^{6}}-\frac{211 u_{4} u_{2}^{2}}{40320 u_{1}^{4}}+\frac{u_{4} u_{2}}{2016 u_{1}^{2}}+\frac{59 u_{3} u_{2}^{4}}{378 u_{1}^{7}} \\
& +\frac{1993 u_{3} u_{2}^{3}}{120960 u_{1}^{5}}-\frac{u_{3} u_{2}^{2}}{576 u_{1}^{3}}-\frac{83 u_{3}^{2} u_{2}^{2}}{896 u_{1}^{6}}+\frac{19 u_{3} u_{2}}{120960 u_{1}}-\frac{17 u_{3}^{2} u_{2}}{2240 u_{1}^{4}}+\frac{1273 u_{3} u_{4} u_{2}}{40320 u_{1}^{5}} .
\end{aligned}
$$

Conjecture 3.3.2 The genus 4 GUE free energy is given by

$$
\begin{aligned}
& F_{4}\left(u_{1}, \ldots, u_{10}\right) \\
& =\frac{1852 u_{2}^{9}}{1215 u_{1}^{12}}+\frac{151 u_{2}^{8}}{675 u_{1}^{10}}-\frac{101 u_{2}^{7}}{12600 u_{1}^{8}}-\frac{772 u_{3} u_{2}^{7}}{135 u_{1}^{11}} \\
& +\frac{9904 u_{4} u_{2}^{6}}{6075 u_{1}^{10}}-\frac{1165 u_{2}^{6}}{1161216 u_{1}^{6}}-\frac{2851 u_{3} u_{2}^{6}}{3600 u_{1}^{9}}+\frac{14903 u_{3}^{2} u_{2}^{5}}{2160 u_{1}^{10}}+\frac{70261 u_{3} u_{2}^{5}}{3225600 u_{1}^{7}} \\
& +\frac{2573 u_{4} u_{2}^{5}}{10800 u_{1}^{8}}+\frac{u_{2}^{5}}{7200 u_{1}^{4}}-\frac{2243 u_{5} u_{2}^{5}}{6480 u_{1}^{9}}+\frac{195677 u_{3}^{2} u_{2}^{4}}{230400 u_{1}^{8}}+\frac{3197 u_{3} u_{2}^{4}}{967680 u_{1}^{5}} \\
& +\frac{12907 u_{6} u_{2}^{4}}{226800 u_{1}^{8}}-\frac{10259 u_{4} u_{2}^{4}}{1935360 u_{1}^{6}}-\frac{22153 u_{5} u_{2}^{4}}{414720 u_{1}^{7}}-\frac{101503 u_{3} u_{4} u_{2}^{4}}{32400 u_{1}^{9}}+\frac{1823 u_{4}^{2} u_{2}^{3}}{5670 u_{1}^{8}} \\
& +\frac{415273 u_{3} u_{5} u_{2}^{3}}{829440 u_{1}^{8}}+\frac{97 u_{5} u_{2}^{3}}{120960 u_{1}^{5}}+\frac{26879 u_{6} u_{2}^{3}}{2903040 u_{1}^{6}}+\frac{u_{2}^{3}}{7257600}-\frac{49 u_{3} u_{2}^{3}}{138240 u_{1}^{3}} \\
& -\frac{5137 u_{4} u_{2}^{3}}{4354560 u_{1}^{4}}-\frac{877 u_{3}^{2} u_{2}^{3}}{57600 u_{1}^{6}}-\frac{812729 u_{3} u_{4} u_{2}^{3}}{2073600 u_{1}^{7}}-\frac{212267 u_{7} u_{2}^{3}}{29030400 u_{1}^{7}}-\frac{305129 u_{3}^{3} u_{2}^{3}}{103680 u_{1}^{9}} \\
& +\frac{u_{1}^{2} u_{2}^{2}}{460800}+\frac{1379 u_{4}^{2} u_{2}^{2}}{34560 u_{1}^{6}}+\frac{13138507 u_{3}^{2} u_{4} u_{2}^{2}}{9676800 u_{1}^{8}}+\frac{2417 u_{3} u_{4} u_{2}^{2}}{537600 u_{1}^{5}}+\frac{17 u_{4} u_{2}^{2}}{138240 u_{1}^{2}} \\
& +\frac{2143 u_{3} u_{5} u_{2}^{2}}{34560 u_{1}^{6}}+\frac{449 u_{5} u_{2}^{2}}{1451520 u_{1}^{3}}+\frac{2323 u_{8} u_{2}^{2}}{3225600 u_{1}^{6}}-\frac{2623 u_{3}^{2} u_{2}^{2}}{967680 u_{1}^{4}}-\frac{443 u_{6} u_{2}^{2}}{9676800 u_{1}^{4}} \\
& -\frac{667 u_{7} u_{2}^{2}}{537600 u_{1}^{5}}-\frac{192983 u_{3}^{3} u_{2}^{2}}{691200 u_{1}^{7}}-\frac{60941 u_{3} u_{6} u_{2}^{2}}{1075200 u_{1}^{7}}-\frac{171343 u_{4} u_{5} u_{2}^{2}}{1935360 u_{1}^{7}}+\frac{22809 u_{3}^{4} u_{2}}{71680 u_{1}^{8}} \\
& +\frac{1747 u_{3}^{3} u_{2}}{806400 u_{1}^{5}}+\frac{7 u_{3}^{2} u_{2}}{38400 u_{1}^{2}}+\frac{9221 u_{5}^{2} u_{2}}{1935360 u_{1}^{6}}+\frac{17 u_{1} u_{3} u_{2}}{3225600}+\frac{78533 u_{3}^{2} u_{4} u_{2}}{691200 u_{1}^{6}} \\
& +\frac{18713 u_{3} u_{4} u_{2}}{14515200 u_{1}^{3}}+\frac{15179 u_{4} u_{6} u_{2}}{1935360 u_{1}^{6}}+\frac{20639 u_{3} u_{7} u_{2}}{4838400 u_{1}^{6}}+\frac{37 u_{8} u_{2}}{302400 u_{1}^{4}}-\frac{u_{4} u_{2}}{86400} \\
& -\frac{11 u_{5} u_{2}}{362880 u_{1}}-\frac{923 u_{6} u_{2}}{14515200 u_{1}^{2}}-\frac{113 u_{7} u_{2}}{9676800 u_{1}^{3}}-\frac{55 u_{4}^{2} u_{2}}{387072 u_{1}^{4}}-\frac{419 u_{3} u_{5} u_{2}}{1935360 u_{1}^{4}}
\end{aligned}
$$




$$
\begin{aligned}
& -\frac{1411 u_{4} u_{5} u_{2}}{138240 u_{1}^{5}}-\frac{7 u_{9} u_{2}}{138240 u_{1}^{5}}-\frac{1751 u_{3} u_{6} u_{2}}{268800 u_{1}^{5}}-\frac{12035 u_{3}^{2} u_{5} u_{2}}{96768 u_{1}^{7}}-\frac{44201 u_{3} u_{4}^{2} u_{2}}{276480 u_{1}^{7}} \\
& +\frac{1549 u_{3}^{4}}{115200 u_{1}^{6}}+\frac{937 u_{3}^{3}}{2903040 u_{1}^{3}}+\frac{229 u_{4}^{3}}{62208 u_{1}^{6}}+\frac{19 u_{5}^{2}}{46080 u_{1}^{4}}+\frac{u_{1}^{3} u_{3}}{691200}+\frac{949 u_{3} u_{4} u_{5}}{55296 u_{1}^{6}} \\
& +\frac{59 u_{3}^{2} u_{6}}{10752 u_{1}^{6}}+\frac{73 u_{4} u_{6}}{107520 u_{1}^{4}}+\frac{1777 u_{3} u_{7}}{4838400 u_{1}^{4}}+\frac{143 u_{7}}{14515200 u_{1}}+\frac{31 u_{8}}{9676800 u_{1}^{2}}+\frac{u_{10}}{497664 u_{1}^{4}} \\
& -\frac{u_{3}^{2}}{115200}-\frac{u_{1} u_{5}}{138240}-\frac{73 u_{6}}{29030400}-\frac{u_{1}^{6}}{43545600}-\frac{19 u_{1}^{2} u_{4}}{87091200}-\frac{137 u_{3} u_{4}}{2073600 u_{1}} \\
& -\frac{239 u_{3} u_{5}}{1451520 u_{1}^{2}}-\frac{661 u_{4}^{2}}{5806080 u_{1}^{2}}-\frac{u_{9}}{138240 u_{1}^{3}}-\frac{17 u_{4} u_{5}}{387072 u_{1}^{3}}-\frac{89 u_{3} u_{6}}{3225600 u_{1}^{3}}-\frac{709 u_{3}^{2} u_{4}}{3225600 u_{1}^{4}} \\
& -\frac{1291 u_{3} u_{4}^{2}}{138240 u_{1}^{5}}-\frac{1001 u_{3}^{2} u_{5}}{138240 u_{1}^{5}}-\frac{197 u_{5} u_{6}}{387072 u_{1}^{5}}-\frac{163 u_{3} u_{8}}{967680 u_{1}^{5}}-\frac{2069 u_{4} u_{7}}{5806080 u_{1}^{5}}-\frac{2153 u_{3}^{3} u_{4}}{28800 u_{1}^{7}}
\end{aligned}
$$

We also computed the genus 5 free energy; see in Appendix A.

For the particular examples of enumerating squares, hexagons, octagons on a genus $g$ surface, one can use (3.3.1), (3.3.2), (A.0.3) to obtain the combinatorial numbers. We checked that these numbers agree with those in [8]. This gives some evidences of validity of the Main Conjecture for $g=3,4,5$.

Remark 3.3.3 The genus 1,2,3 terms of the GUE free energy with even couplings were also derived in [13, 14, 26] for the particular case of only one nonzero coupling (i.e., in the framework of enumeration of 2m-gons). To the best of our knowledge, explicit formulae for higher genus $(g \geq 4)$ terms, even in the case of the particular examples, were not available in the literature.

\section{A Explicit formula for $F_{5}$}

$$
\begin{aligned}
& F_{5}\left(u_{1}, \ldots, u_{13}\right)= \\
& \quad-\frac{109514 u_{2}^{12}}{1215 u_{1}^{16}}-\frac{1352 u_{2}^{11}}{81 u_{1}^{14}}+\frac{181628 u_{3} u_{2}^{10}}{405 u_{1}^{15}}-\frac{2593 u_{2}^{10}}{9450 u_{1}^{12}}+\frac{42691 u_{3} u_{2}^{9}}{540 u_{1}^{13}} \\
&+\frac{16091 u_{2}^{9}}{187110 u_{1}^{10}}-\frac{93460 u_{4} u_{2}^{9}}{729 u_{1}^{14}}+\frac{600763 u_{3} u_{2}^{8}}{415800 u_{1}^{11}}+\frac{134599 u_{5} u_{2}^{8}}{4860 u_{1}^{13}}-\frac{274289 u_{2}^{8}}{255467520 u_{1}^{8}} \\
&-\frac{567199 u_{4} u_{2}^{8}}{24300 u_{1}^{12}}-\frac{1322159 u_{3}^{2} u_{2}^{8}}{1620 u_{1}^{14}}+\frac{391519 u_{3} u_{4} u_{2}^{7}}{972 u_{1}^{13}}+\frac{2471441 u_{5} u_{2}^{7}}{475200 u_{1}^{11}}-\frac{151 u_{2}^{7}}{399168 u_{1}^{6}} \\
&-\frac{999473 u_{3} u_{2}^{7}}{2838528 u_{1}^{9}}-\frac{2825 u_{4} u_{2}^{7}}{5544 u_{1}^{10}}-\frac{1149739 u_{3}^{2} u_{2}^{7}}{8640 u_{1}^{12}}-\frac{161353 u_{6} u_{2}^{7}}{34020 u_{1}^{12}}+\frac{12916717 u_{3}^{3} u_{2}^{6}}{19440 u_{1}^{13}} \\
&+\frac{319877 u_{3} u_{2}^{6}}{159667200 u_{1}^{7}}+\frac{31781177 u_{3} u_{4} u_{2}^{6}}{475200 u_{1}^{11}}+\frac{4549471 u_{4} u_{2}^{6}}{42577920 u_{1}^{8}}+\frac{515032871 u_{5} u_{2}^{6}}{3832012800 u_{1}^{9}} \\
&+ \\
&+\frac{8477461 u_{7} u_{2}^{6}}{12830400 u_{1}^{11}}+\frac{263 u_{2}^{6}}{23950080 u_{1}^{4}}-\frac{27484783 u_{6} u_{2}^{6}}{29937600 u_{1}^{10}}-\frac{1098923921 u_{3}^{2} u_{2}^{6}}{425779200 u_{1}^{10}} \\
&- \frac{5713573 u_{3} u_{5} u_{2}^{6}}{77760 u_{1}^{12}}-\frac{12051881 u_{4}^{2} u_{2}^{6}}{255150 u_{1}^{12}}+\frac{173831501 u_{3}^{3} u_{2}^{5}}{1824768 u_{1}^{11}}+\frac{6013615 u_{3}^{2} u_{2}^{5}}{12773376 u_{1}^{8}}
\end{aligned}
$$




$$
\begin{aligned}
& +\frac{28957 u_{3} u_{2}^{5}}{21288960 u_{1}^{5}}+\frac{276125491 u_{3} u_{4} u_{2}^{5}}{182476800 u_{1}^{9}}+\frac{10243 u_{4} u_{2}^{5}}{239500800 u_{1}^{6}}+\frac{53761259 u_{4} u_{5} u_{2}^{5}}{3326400 u_{1}^{11}} \\
& +\frac{102985067 u_{3} u_{6} u_{2}^{5}}{9979200 u_{1}^{11}}+\frac{84091529 u_{7} u_{2}^{5}}{638668800 u_{1}^{9}}+\frac{u_{2}^{5}}{633600 u_{1}^{2}}-\frac{11532541 u_{5} u_{2}^{5}}{479001600 u_{1}^{7}}-\frac{5117003 u_{6} u_{2}^{5}}{182476800 u_{1}^{8}} \\
& -\frac{19847231 u_{4}^{2} u_{2}^{5}}{2494800 u_{1}^{10}}-\frac{2268769 u_{8} u_{2}^{5}}{29937600 u_{1}^{10}}-\frac{1125727817 u_{3} u_{5} u_{2}^{5}}{91238400 u_{1}^{10}}-\frac{14531719 u_{3}^{2} u_{4} u_{2}^{5}}{36288 u_{1}^{12}} \\
& +\frac{2322129119 u_{3}^{3} u_{2}^{4}}{1277337600 u_{1}^{9}}+\frac{1211 u_{3}^{2} u_{2}^{4}}{2027520 u_{1}^{6}}+\frac{87822499 u_{3} u_{4}^{2} u_{2}^{4}}{1197504 u_{1}^{11}}+\frac{728671781 u_{3}^{2} u_{5} u_{2}^{4}}{12773376 u_{1}^{11}} \\
& +\frac{1046529431 u_{4} u_{5} u_{2}^{4}}{383201280 u_{1}^{9}}+\frac{1108533611 u_{3} u_{6} u_{2}^{4}}{638668800 u_{1}^{9}}+\frac{231617 u_{6} u_{2}^{4}}{54743040 u_{1}^{6}}+\frac{1215667 u_{7} u_{2}^{4}}{255467520 u_{1}^{7}}+\frac{1096859 u_{9} u_{2}^{4}}{153280512 u_{1}^{9}} \\
& -\frac{29 u_{2}^{4}}{127733760}-\frac{47 u_{3} u_{2}^{4}}{1596672 u_{1}^{3}}-\frac{29783 u_{4} u_{2}^{4}}{63866880 u_{1}^{4}}-\frac{24971 u_{5} u_{2}^{4}}{127733760 u_{1}^{5}}-\frac{443511251 u_{3} u_{4} u_{2}^{4}}{1916006400 u_{1}^{7}} \\
& -\frac{68301953 u_{3} u_{5} u_{2}^{4}}{212889600 u_{1}^{8}}-\frac{5935327 u_{8} u_{2}^{4}}{383201280 u_{1}^{8}}-\frac{133150489 u_{4}^{2} u_{2}^{4}}{638668800 u_{1}^{8}}-\frac{5682077 u_{4} u_{6} u_{2}^{4}}{2721600 u_{1}^{10}} \\
& -\frac{1095679399 u_{3}^{2} u_{4} u_{2}^{4}}{19353600 u_{1}^{10}}-\frac{154991051 u_{3} u_{7} u_{2}^{4}}{136857600 u_{1}^{10}}-\frac{4872743377 u_{5}^{2} u_{2}^{4}}{3832012800 u_{1}^{10}}-\frac{547560589 u_{3}^{4} u_{2}^{4}}{2322432 u_{1}^{12}} \\
& +\frac{u_{1}^{2} u_{2}^{3}}{3991680}+\frac{69761129 u_{3} u_{4}^{2} u_{2}^{3}}{6967296 u_{1}^{9}}+\frac{75292039 u_{4}^{2} u_{2}^{3}}{2874009600 u_{1}^{6}}+\frac{8817996169 u_{3}^{3} u_{4} u_{2}^{3}}{63866880 u_{1}^{11}} \\
& +\frac{1049 u_{4} u_{2}^{3}}{119750400 u_{1}^{2}}+\frac{2479931059 u_{3}^{2} u_{5} u_{2}^{3}}{319334400 u_{1}^{9}}+\frac{38370113 u_{3} u_{5} u_{2}^{3}}{958003200 u_{1}^{6}}+\frac{51887531 u_{4} u_{5} u_{2}^{3}}{638668800 u_{1}^{7}} \\
& +\frac{2279 u_{5} u_{2}^{3}}{19160064 u_{1}^{3}}+\frac{16316161 u_{3} u_{6} u_{2}^{3}}{319334400 u_{1}^{7}}+\frac{25527371 u_{5} u_{6} u_{2}^{3}}{87091200 u_{1}^{9}}+\frac{6169 u_{6} u_{2}^{3}}{76640256 u_{1}^{4}}+\frac{791123 u_{4} u_{7} u_{2}^{3}}{3870720 u_{1}^{9}} \\
& +\frac{369691943 u_{3} u_{8} u_{2}^{3}}{3832012800 u_{1}^{9}}+\frac{284539 u_{9} u_{2}^{3}}{191600640 u_{1}^{7}}-\frac{767 u_{3} u_{2}^{3}}{212889600 u_{1}}-\frac{2141 u_{3}^{2} u_{2}^{3}}{1520640 u_{1}^{4}}-\frac{42149 u_{7} u_{2}^{3}}{71850240 u_{1}^{5}} \\
& -\frac{1229917 u_{3} u_{4} u_{2}^{3}}{638668800 u_{1}^{5}}-\frac{52781 u_{8} u_{2}^{3}}{79833600 u_{1}^{6}}-\frac{10864759 u_{3}^{3} u_{2}^{3}}{47900160 u_{1}^{7}}-\frac{2883761 u_{4} u_{6} u_{2}^{3}}{8294400 u_{1}^{8}} \\
& -\frac{764936639 u_{3}^{2} u_{4} u_{2}^{3}}{638668800 u_{1}^{8}}-\frac{179669059 u_{3} u_{7} u_{2}^{3}}{958003200 u_{1}^{8}}-\frac{573019 u_{10} u_{2}^{3}}{1045094400 u_{1}^{8}}-\frac{406451147 u_{5}^{2} u_{2}^{3}}{1916006400 u_{1}^{8}} \\
& -\frac{1848263 u_{4}^{3} u_{2}^{3}}{467775 u_{1}^{10}}-\frac{626921467 u_{3}^{2} u_{6} u_{2}^{3}}{106444800 u_{1}^{10}}-\frac{3430962641 u_{3}^{4} u_{2}^{3}}{127733760 u_{1}^{10}}-\frac{35338084651 u_{3} u_{4} u_{5} u_{2}^{3}}{1916006400 u_{1}^{10}} \\
& +\frac{419193 u_{3}^{5} u_{2}^{2}}{14336 u_{1}^{11}}+\frac{u_{1}^{4} u_{2}^{2}}{6386688}+\frac{23669 u_{3}^{2} u_{2}^{2}}{1277337600 u_{1}^{2}}+\frac{1783923 u_{3} u_{4}^{2} u_{2}^{2}}{7884800 u_{1}^{7}}+\frac{50047 u_{4}^{2} u_{2}^{2}}{106444800 u_{1}^{4}} \\
& +\frac{1238492531 u_{3} u_{5}^{2} u_{2}^{2}}{1277337600 u_{1}^{9}}+\frac{181880015 u_{3}^{3} u_{4} u_{2}^{2}}{12773376 u_{1}^{9}}+\frac{73978651 u_{3}^{2} u_{4} u_{2}^{2}}{638668800 u_{1}^{6}}+\frac{3337 u_{3} u_{4} u_{2}^{2}}{4561920 u_{1}^{3}} \\
& +\frac{5302619 u_{3}^{2} u_{5} u_{2}^{2}}{30412800 u_{1}^{7}}+\frac{1591687897 u_{4}^{2} u_{5} u_{2}^{2}}{1277337600 u_{1}^{9}}+\frac{30953 u_{3} u_{5} u_{2}^{2}}{45619200 u_{1}^{4}}+\frac{203383903 u_{3} u_{4} u_{6} u_{2}^{2}}{127733760 u_{1}^{9}} \\
& +\frac{29758507 u_{5} u_{6} u_{2}^{2}}{638668800 u_{1}^{7}}+\frac{275874283 u_{3}^{2} u_{7} u_{2}^{2}}{638668800 u_{1}^{9}}+\frac{2069131 u_{4} u_{7} u_{2}^{2}}{63866880 u_{1}^{7}}+\frac{289 u_{3} u_{8} u_{2}^{2}}{19008 u_{1}^{7}}+\frac{60127 u_{8} u_{2}^{2}}{958003200 u_{1}^{4}} \\
& +\frac{3373 u_{9} u_{2}^{2}}{45619200 u_{1}^{5}}+\frac{11437 u_{11} u_{2}^{2}}{348364800 u_{1}^{7}}-\frac{5 u_{1} u_{3} u_{2}^{2}}{2128896}-\frac{19 u_{4} u_{2}^{2}}{54743040}-\frac{7753 u_{5} u_{2}^{2}}{3832012800 u_{1}}-\frac{223 u_{6} u_{2}^{2}}{9123840 u_{1}^{2}}
\end{aligned}
$$




$$
\begin{aligned}
& -\frac{247 u_{7} u_{2}^{2}}{11612160 u_{1}^{3}}-\frac{317077 u_{3} u_{6} u_{2}^{2}}{63866880 u_{1}^{5}}-\frac{998201 u_{3}^{3} u_{2}^{2}}{638668800 u_{1}^{5}}-\frac{5091301 u_{4} u_{5} u_{2}^{2}}{638668800 u_{1}^{5}}-\frac{742733 u_{5}^{2} u_{2}^{2}}{106444800 u_{1}^{6}} \\
& -\frac{19631 u_{10} u_{2}^{2}}{174182400 u_{1}^{6}}-\frac{1295627 u_{3} u_{7} u_{2}^{2}}{212889600 u_{1}^{6}}-\frac{7277719 u_{4} u_{6} u_{2}^{2}}{638668800 u_{1}^{6}}-\frac{20664649 u_{3} u_{4} u_{5} u_{2}^{2}}{8870400 u_{1}^{8}}-\frac{9854357 u_{3}^{2} u_{6} u_{2}^{2}}{13305600 u_{1}^{8}} \\
& -\frac{13316641 u_{4}^{3} u_{2}^{2}}{26611200 u_{1}^{8}}-\frac{1293697 u_{5} u_{7} u_{2}^{2}}{53222400 u_{1}^{8}}-\frac{328171 u_{3} u_{9} u_{2}^{2}}{53222400 u_{1}^{8}}-\frac{45787691 u_{3}^{4} u_{2}^{2}}{106444800 u_{1}^{8}}-\frac{6093371 u_{6}^{2} u_{2}^{2}}{425779200 u_{1}^{8}} \\
& -\frac{18837199 u_{4} u_{8} u_{2}^{2}}{1277337600 u_{1}^{8}}-\frac{2912894597 u_{3}^{2} u_{4}^{2} u_{2}^{2}}{116121600 u_{1}^{10}}-\frac{1661611993 u_{3}^{3} u_{5} u_{2}^{2}}{127733760 u_{1}^{10}} \\
& +\frac{14367497 u_{3}^{5} u_{2}}{7096320 u_{1}^{9}}+\frac{3625841 u_{3}^{4} u_{2}}{127733760 u_{1}^{6}}+\frac{4721 u_{3}^{3} u_{2}}{12773376 u_{1}^{3}}+\frac{274614007 u_{3} u_{4}^{3} u_{2}}{182476800 u_{1}^{9}}+\frac{7368997 u_{3} u_{5}^{2} u_{2}}{70963200 u_{1}^{7}} \\
& +\frac{927517 u_{5}^{2} u_{2}}{1916006400 u_{1}^{4}}+\frac{17 u_{1}^{3} u_{3} u_{2}}{53222400}+\frac{2872733 u_{3}^{3} u_{4} u_{2}}{13305600 u_{1}^{7}}+\frac{20527 u_{3}^{2} u_{4} u_{2}}{14192640 u_{1}^{4}}+\frac{85455011 u_{4}^{2} u_{5} u_{2}}{638668800 u_{1}^{7}} \\
& +\frac{1121508611 u_{3}^{2} u_{4} u_{5} u_{2}}{319334400 u_{1}^{9}}+\frac{159183659 u_{3}^{3} u_{6} u_{2}}{212889600 u_{1}^{9}}+\frac{15535133 u_{3} u_{4} u_{6} u_{2}}{91238400 u_{1}^{7}}+\frac{1516703 u_{4} u_{6} u_{2}}{1916006400 u_{1}^{4}} \\
& +\frac{2867 u_{5} u_{6} u_{2}}{1814400 u_{1}^{5}}+\frac{5833 u_{6} u_{2}}{3832012800}+\frac{916913 u_{3}^{2} u_{7} u_{2}}{19958400 u_{1}^{7}}+\frac{49661 u_{3} u_{7} u_{2}}{119750400 u_{1}^{4}}+\frac{698809 u_{4} u_{7} u_{2}}{638668800 u_{1}^{5}} \\
& +\frac{82861 u_{6} u_{7} u_{2}}{45619200 u_{1}^{7}}+\frac{373 u_{7} u_{2}}{91238400 u_{1}}+\frac{650429 u_{3} u_{8} u_{2}}{1277337600 u_{1}^{5}}+\frac{122413 u_{5} u_{8} u_{2}}{91238400 u_{1}^{7}}+\frac{41 u_{8} u_{2}}{9580032 u_{1}^{2}} \\
& +\frac{38377 u_{4} u_{9} u_{2}}{53222400 u_{1}^{7}}+\frac{3929 u_{3} u_{10} u_{2}}{14515200 u_{1}^{7}}+\frac{1091 u_{11} u_{2}}{174182400 u_{1}^{5}}-\frac{19 u_{1} u_{5} u_{2}}{13305600}-\frac{23 u_{1}^{2} u_{4} u_{2}}{15966720}-\frac{23 u_{3}^{2} u_{2}}{21288960} \\
& -\frac{4801 u_{3} u_{4} u_{2}}{766402560 u_{1}}-\frac{5497 u_{4}^{2} u_{2}}{63866880 u_{1}^{2}}-\frac{26833 u_{3} u_{5} u_{2}}{212889600 u_{1}^{2}}-\frac{323 u_{9} u_{2}}{68428800 u_{1}^{3}}-\frac{10961 u_{3} u_{6} u_{2}}{79833600 u_{1}^{3}}-\frac{64907 u_{4} u_{5} u_{2}}{273715200 u_{1}^{3}} \\
& -\frac{727 u_{10} u_{2}}{116121600 u_{1}^{4}}-\frac{1883363 u_{3}^{2} u_{5} u_{2}}{159667200 u_{1}^{5}}-\frac{3705967 u_{3} u_{4}^{2} u_{2}}{239500800 u_{1}^{5}}-\frac{7 u_{12} u_{2}}{4976640 u_{1}^{6}}-\frac{14227 u_{6}^{2} u_{2}}{7096320 u_{1}^{6}}-\frac{16217 u_{4} u_{8} u_{2}}{7884800 u_{1}^{6}} \\
& -\frac{13001 u_{3} u_{9} u_{2}}{15206400 u_{1}^{6}}-\frac{864373 u_{3}^{2} u_{6} u_{2}}{53222400 u_{1}^{6}}-\frac{1184501 u_{4}^{3} u_{2}}{106444800 u_{1}^{6}}-\frac{542981 u_{5} u_{7} u_{2}}{159667200 u_{1}^{6}}-\frac{32913731 u_{3} u_{4} u_{5} u_{2}}{638668800 u_{1}^{6}} \\
& -\frac{1144789 u_{4} u_{5}^{2} u_{2}}{11612160 u_{1}^{8}}-\frac{946477 u_{3}^{2} u_{8} u_{2}}{45619200 u_{1}^{8}}-\frac{176093453 u_{3}^{3} u_{5} u_{2}}{159667200 u_{1}^{8}}-\frac{454075417 u_{3}^{2} u_{4}^{2} u_{2}}{212889600 u_{1}^{8}}-\frac{28069627 u_{3} u_{4} u_{7} u_{2}}{319334400 u_{1}^{8}} \\
& -\frac{80480347 u_{3} u_{5} u_{6} u_{2}}{638668800 u_{1}^{8}}-\frac{103432013 u_{4}^{2} u_{6} u_{2}}{1277337600 u_{1}^{8}}-\frac{18599541 u_{3}^{4} u_{4} u_{2}}{1576960 u_{1}^{10}}+\frac{10673 u_{3}^{5}}{691200 u_{1}^{7}}+\frac{16649 u_{3}^{4}}{91238400 u_{1}^{4}} \\
& +\frac{275599 u_{3} u_{4}^{3}}{3379200 u_{1}^{7}}+\frac{800453 u_{4}^{3}}{1916006400 u_{1}^{4}}+\frac{571213 u_{5}^{3}}{383201280 u_{1}^{7}}+\frac{64482661 u_{3}^{3} u_{4}^{2}}{60825600 u_{1}^{9}}+\frac{1217 u_{4}^{2}}{1149603840}+\frac{2264879 u_{3} u_{5}^{2}}{1277337600 u_{1}^{5}} \\
& +\frac{7003 u_{5}^{2}}{348364800 u_{1}^{2}}+\frac{66653 u_{3} u_{6}^{2}}{28385280 u_{1}^{7}}+\frac{u_{1}^{5} u_{3}}{15966720}+\frac{35113699 u_{3}^{4} u_{5}}{85155840 u_{1}^{9}}+\frac{2932793 u_{4}^{2} u_{5}}{1277337600 u_{1}^{5}}+\frac{4643 u_{3} u_{5}}{1916006400} \\
& +\frac{30303149 u_{3}^{2} u_{4} u_{5}}{159667200 u_{1}^{7}}+\frac{18211 u_{3} u_{4} u_{5}}{9580032 u_{1}^{4}}+\frac{1867 u_{4} u_{5}}{79833600 u_{1}}+\frac{570989 u_{3}^{3} u_{6}}{14192640 u_{1}^{7}}+\frac{13 u_{1}^{2} u_{6}}{174182400}+\frac{62701 u_{3}^{2} u_{6}}{106444800 u_{1}^{4}} \\
& +\frac{8669 u_{3} u_{6}}{638668800 u_{1}}+\frac{102911 u_{3} u_{4} u_{6}}{35481600 u_{1}^{5}}+\frac{20521 u_{4} u_{6}}{638668800 u_{1}^{2}}+\frac{104281 u_{4} u_{5} u_{6}}{14192640 u_{1}^{7}}+\frac{4717 u_{3}^{2} u_{7}}{6082560 u_{1}^{5}} \\
& +\frac{155959 u_{4}^{2} u_{7}}{60825600 u_{1}^{7}}+\frac{6911 u_{1} u_{7}}{11496038400}+\frac{29611 u_{3} u_{7}}{1916006400 u_{1}^{2}}+\frac{318517 u_{3} u_{5} u_{7}}{79833600 u_{1}^{7}}+\frac{299 u_{6} u_{7}}{1689600 u_{1}^{5}}+\frac{1030877 u_{3} u_{4} u_{8}}{425779200 u_{1}^{7}}
\end{aligned}
$$




$$
\begin{aligned}
& +\frac{31 u_{5} u_{8}}{237600 u_{1}^{5}}+\frac{7697 u_{3}^{2} u_{9}}{15206400 u_{1}^{7}}+\frac{44647 u_{4} u_{9}}{638668800 u_{1}^{5}}+\frac{71 u_{3} u_{10}}{2721600 u_{1}^{5}}+\frac{13 u_{10}}{74649600 u_{1}^{2}}+\frac{113 u_{11}}{348364800 u_{1}^{3}} \\
& +\frac{u_{13}}{29859840 u_{1}^{5}}-\frac{23 u_{1}^{2} u_{3}^{2}}{21288960}-\frac{u_{1}^{4} u_{4}}{130636800}-\frac{2011 u_{1} u_{3} u_{4}}{638668800}-\frac{79 u_{8}}{638668800}-\frac{241 u_{1}^{3} u_{5}}{958003200}-\frac{u_{1}^{8}}{1277337600} \\
& -\frac{361 u_{9}}{547430400 u_{1}}-\frac{5707 u_{3}^{3}}{3832012800 u_{1}}-\frac{2099 u_{3}^{2} u_{4}}{15966720 u_{1}^{2}}-\frac{931 u_{4} u_{7}}{22809600 u_{1}^{3}}-\frac{13261 u_{3} u_{4}^{2}}{54743040 u_{1}^{3}}-\frac{10277 u_{3}^{2} u_{5}}{58060800 u_{1}^{3}} \\
& -\frac{17527 u_{3} u_{8}}{958003200 u_{1}^{3}}-\frac{113089 u_{5} u_{6}}{1916006400 u_{1}^{3}}-\frac{u_{12}}{4976640 u_{1}^{4}}-\frac{2533 u_{3} u_{9}}{106444800 u_{1}^{4}}-\frac{8017 u_{6}^{2}}{141926400 u_{1}^{4}}-\frac{8189 u_{4} u_{8}}{141926400 u_{1}^{4}} \\
& -\frac{30553 u_{5} u_{7}}{319334400 u_{1}^{4}}-\frac{809239 u_{3}^{3} u_{4}}{106444800 u_{1}^{5}}-\frac{11 u_{3} u_{11}}{1741824 u_{1}^{6}}-\frac{22349 u_{4} u_{5}^{2}}{3193344 u_{1}^{6}}-\frac{701 u_{5} u_{9}}{18247680 u_{1}^{6}}-\frac{2143 u_{6} u_{8}}{36495360 u_{1}^{6}} \\
& -\frac{775 u_{4} u_{10}}{41803776 u_{1}^{6}}-\frac{520931 u_{3}^{3} u_{5}}{42577920 u_{1}^{6}}-\frac{244457 u_{4}^{2} u_{6}}{42577920 u_{1}^{6}}-\frac{659977 u_{3} u_{4} u_{7}}{106444800 u_{1}^{6}}-\frac{3697 u_{7}^{2}}{109486080 u_{1}^{6}}-\frac{1138241 u_{3} u_{5} u_{6}}{127733760 u_{1}^{6}} \\
& -\frac{185851 u_{3}^{2} u_{8}}{127733760 u_{1}^{6}}-\frac{10135361 u_{3}^{2} u_{4}^{2}}{425779200 u_{1}^{6}}-\frac{336827 u_{3}^{2} u_{4} u_{6}}{2956800 u_{1}^{8}}-\frac{3980637 u_{3}^{4} u_{4}}{7884800 u_{1}^{8}}-\frac{653701 u_{4}^{4}}{34214400 u_{1}^{8}} \\
& -\frac{877403 u_{3}^{3} u_{7}}{42577920 u_{1}^{8}}-\frac{8134913 u_{3} u_{4}^{2} u_{5}}{45619200 u_{1}^{8}}-\frac{22151509 u_{3}^{2} u_{5}^{2}}{319334400 u_{1}^{8}}-\frac{4543 u_{3}^{6}}{8192 u_{1}^{10} .}
\end{aligned}
$$

\section{References}

[1] Bessis, D., Itzykson, C., Zuber, J. B. (1980). Quantum field theory techniques in graphical enumeration. Advances in Applied Mathematics, 1 (2), 109-157.

[2] Brézin, E., Itzykson, C., Parisi, P., Zuber, J.-B. (1978). Planar diagrams. Commun. Math. Phys., $\mathbf{5 9}, 35-51$.

[3] Buryak, A. (2013). Dubrovin-Zhang hierarchy for the Hodge integrals. Commun. Number Theory and Physics, 9 (2015) 239-271. Preprint arXiv: 1308.5716.

[4] Carlet, G., Dubrovin, B., Zhang, Y. (2004). The extended Toda hierarchy. Mosc. Math. J., 4 (2), 313-332.

[5] Dijkgraaf, R., Witten, E. (1990) Mean field theory, topological field theory, and multi-matrix models, Nucl. Phys. B 342 (1990) 486-522.

[6] Dubrovin, B. (1996). Geometry of 2D topological field theories. In "Integrable Systems and Quantum Groups", Editors: Francaviglia, M., Greco, S. Springer Lecture Notes in Math. 1620, 120348.

[7] Dubrovin, B. (2009). Hamiltonian perturbations of hyperbolic PDEs: from classification results to the properties of solutions. In: New trends in in Mathematical Physics. Selected contributions of the XVth International Congress on Mathematical Physics. Sidoravicius, Vladas (Ed.), Springer Netherlands, 231-276.

[8] Dubrovin, B., Yang, D. (2016). Generating series for GUE correlators. Preprint arXiv: 1604.07628. 
[9] Dubrovin, B., Yang, D. Remarks on intersection numbers and integrable hierarchies. I. Quasitriviality. to appear.

[10] Dubrovin, B., Zhang, Y. (2001). Normal forms of hierarchies of integrable PDEs, Frobenius manifolds and Gromov-Witten invariants. Preprint arXiv: math.DG/0108160.

[11] Dubrovin, B., Zhang, Y. (2004). Virasoro symmetries of the extended Toda hierarchy. Comm. Math. Phys., 250 (1), 161-193.

[12] Dubrovin, B., Liu, S.-Q., Yang, D., Zhang, Y. (2016). Hodge integrals and tau-symmetric integrable hierarchies of Hamiltonian evolutionary PDEs. Advances in Mathematics, 293, 382-435.

[13] Ercolani, N. M. (2011). Caustics, counting maps and semi-classical asymptotics. Nonlinearity, 24 (2), 481-526.

[14] Ercolani, N. M., McLaughlin, K. D. T.-R., Pierce, V. U. (2008). Random matrices, graphical enumeration and the continuum limit of the Toda lattices. Comm. Math. Phys., 278 (1), 31-81.

[15] Ercolani, N. M., Pierce, V. U. (2012). The continuum limit of Toda lattices for random matrices with odd weights. Comm. Math. Sci. 10, 267-305.

[16] Faber, C., Pandharipande, R. (2000). Hodge integrals and Gromov-Witten theory. Inventiones mathematicae, 139 (1), 173-199.

[17] Gopakumar, R., Vafa, C. (1999). On the gauge theory/geometry correspondence, Adv. Theor. Math. Phys., 5, 1415-1443.

[18] Liu, C. C. M., Liu, K., Zhou, J. (2003). A proof of a conjecture of Mariño-Vafa on Hodge integrals. Journal of Differential Geometry, 65 (2), 289-340.

[19] Liu, C. C. M., Liu, K., Zhou, J. (2004). On a proof of a conjecture of Mariño-Vafa on Hodge Integrals. Mathematical Research Letters, 11 (2), 259-272.

[20] Liu, C. C. M., Liu, K., Zhou, J. (2006). Mariño-Vafa formula and Hodge integral identities. Journal of Algebraic Geometry, 15 (2), 379-398.

[21] 't Hooft, G. (1974). A planar diagram theory for strong interactions. Nucl. Phys. B 72, 461-473.

[22] 't Hooft, G. (1974). A two-dimensional model for mesons. Nucl. Phys. B 75, 461-470.

[23] Mehta, M. L. (1991). Random Matrices, 2nd edition, Academic Press, New York.

[24] Mariño, M., Vafa, C. (2002). Framed knots at large N. Contemporary Mathematics, 310, 185-204.

[25] Okounkov, A., Pandharipande, R. (2004). Hodge integrals and invariants of the unknot. Geometry \& Topology, 8 (2), 675-699.

[26] Pierce, V. U. (2013). Continuum limits of Toda lattices for map enumeration. In: Algebraic and Geometric Aspects of Integrable Systems and Random Matrices, edited by Anton Dzhamay, Kenichi Maruno, Virgil U. Pierce; Contemporary Mathematics 593, 1-29.

[27] Witten, E. (1991). Two-dimensional gravity and intersection theory on moduli space. Surveys in Differential Geometry (Cambridge, MA, 1990), (pp. 243-310), Lehigh Univ., Bethlehem, PA. 
[28] Zhou, J. (2010). Hodge integrals and integrable hierarchies. Letters in Mathematical Physics, 93 (1), 55-71.

E-mails: dubrovin@sissa.it, dyang@sissa.it 